\section{TNFRSF1A Gene Causing Tumor Necrosis Factor Receptor-associated Periodic Syndrome in 2 Siblings Displaying Variable Disease Severity and Discordant Heterozygosity for an MEF V E148Q Variant}

\section{To the Editor:}

Tumor necrosis factor receptor-associated periodic syndrome (TRAPS) is an autosomal dominant periodic fever syndrome with pan-ethnic distribution caused by mutations in TNFRSF $1 A^{1}$. To date, over 100 mutations have been described. Among whites, the R92Q mutation is the most frequently observed variant of the TNFRSF1A gene. R92Q is a missense low-penetrance gene mutation that is associated with a mild severity, high rate of spontaneous amelioration, and lower prevalence of amyloidosis compared with structural gene mutation variants ${ }^{2}$. Despite its relatively high prevalence in the general population, ranging from $1.2 \%$ to $4 \%$, only 1 clinically ascertained patient has been described with homozygous R92Q variant $^{3}$. We hereby describe 2 Omani sisters with TRAPS caused by homozygous R92Q variants in TNFRSF1A. One of the siblings also had associated E148Q variant in the $M E F V$ gene. They both displayed severe phenotype with evident clinical variability.

A 12-year-old girl presented at 18 months of age with frequent episodes of high-grade fever lasting between 5 to 14 days occurring at 1-3 monthly intervals. These attacks were associated with severe abdominal pain, vomiting, myalgias, arthralgias, and chest pains. There was no associated skin rash or conjunctivitis. In between the attacks, she was completely well. Investigations during episodes revealed anemia, leukocytosis, thrombocytosis, and elevated inflammatory markers. Sequencing the $M E F V$ gene revealed a heterozygous E148Q in the $M E F V$ gene. She was diagnosed with familial Mediterranean fever (FMF) and started treatment with colchicine. After 4 years, the frequency and severity of attacks increased, prompting etanercept (ETN) to be started. Two years later, the severity and frequency of febrile episodes reoccurred. The diagnosis of TRAPS was then considered when mild periorbital swelling was noted during febrile episodes. Genetic testing revealed a homozygous $\mathrm{R} 92 \mathrm{Q}$ mutation identified in the TNFRSF1A gene, consistent with TRAPS. The patient was treated with anakinra at a dose of $2 \mathrm{mg} / \mathrm{kg} / \mathrm{sc}$ daily with dramatic improvement in clinical and laboratory variables (Table 1). She has not had any further attacks during 2 years of followup.

The patient's 6-year-old sister also presented at the age of 18 months with similar febrile episodes. However, her attacks were much milder in severity and less frequent, occurring at 3-6 monthly intervals. In view of the initial diagnosis of FMF in her sibling, that diagnosis was considered. The patient began treatment with colchicine without significant improvement. She was then treated with ETN with only modest improvement. The diagnosis was reevaluated and the diagnosis of TRAPS was considered. Genetic testing revealed the familial homozygous R92Q mutation identified

Table 1. Effect of anakinra on various laboratory variables in each sibling.

\begin{tabular}{lcccc}
\hline Test & \multicolumn{2}{c}{ Before Anakinra } & \multicolumn{2}{c}{ After Anakinra } \\
& Patient 1 & Patient 2 & Patient 1 & Patient 2 \\
\hline $\begin{array}{l}\text { Hemoglobin, } \\
\quad \text { 11.5-15.5 g/dl }\end{array}$ & 9.0 & 10 & 12.2 & 11.2 \\
$\quad \begin{array}{l}\text { White cell count, } \\
\quad 4.5-14.5 \times 10^{9} / 1\end{array}$ & 17.6 & 10.4 & 6.0 & 5.8 \\
Platelet count, & & & & \\
$\quad 50-450 \times 10^{9} / 1$ & 700 & 986 & 302 & 433 \\
ESR, $1-25 \mathrm{~mm} / \mathrm{h}$ & 102 & 57 & 5 & 14 \\
CRP, <5 mg/l & 283 & 70 & 2 & 5 \\
S-amyloid A protein, & & & & \\
$\quad<15 \mathrm{mg} / 1$ & 1130 & 1140 & 5 & 13 \\
\hline
\end{tabular}

ESR: erythrocyte sedimentation rate; CRP: C-reactive protein. in her sister, but she was not found to have the E148Q variant in $M E F V$. Therefore, anakinra was commenced at a dose of $2 \mathrm{mg} / \mathrm{kg} / \mathrm{sc}$ daily with dramatic improvement in clinical manifestation and laboratory findings, with no recurrence of attacks in 2 years of followup. The completely asymptomatic parents of these 2 siblings were found to be heterozygous for the R92Q variants. The mother was also found to be heterozygous for the E148Q variant in $M E F V$ (Figure 1).

To our knowledge, the patients we describe represent the second reported cases of TRAPS caused by homozygous R92Q variants. The previously described patient showed a milder disease course ${ }^{3}$. Given the frequency of the R92Q variant in the general population, homozygosity for this variant may not be uncommon and one wonders whether the paucity of publications about R92Q homozygosity is because of publication bias, or the result of lack of genetic testing. On the other hand, one cannot exclude that homozygosity for this variant alone is not enough for disease manifestations to occur, and additional genetic and/or environmental factors may be required for phenotypic expression.

The cases we describe suggest that the periodic fever phenotype in these 2 cases has been caused by homozygosity for the R92Q variant in the TNFRSF1A gene. Although this is known to be a mild variant with reduced penetrance in a heterozygous state, one cannot exclude that homozygosity for this variant may result in a more severe phenotype or increase the penetrance, because it is well established in mutations seen in other autosomal dominant disorders when inherited in homozygous state.

The E148Q found in this family has been shown to display features to suggest that it is likely a susceptibility variant for autoinflammation rather than an FMF-causing mutation ${ }^{4,5,6,7}$. It is unlikely that this variant has contributed to the periodic fever syndrome seen in this family. One of the 2 sisters who displayed TRAPS in this family did not have this variant, and her mother has not manifested disease symptoms despite being heterozygous for both variants. The role of this variant as a disease modifier cannot be excluded because the children who were discordant for this variant also showed some clinical variability. The 2 cases we describe highlight the importance of clinical assessment and comprehensive genetic testing when evaluating patients with periodic fever syndromes, particularly in patients displaying atypical response to standard therapy.

REEM ABDWANI, MD, FAAP, FRCPC, FRCPCH; EIMAN ABDALLA, MD, Department of Child Health, Sultan Qaboos University Hospital; SAFIYA AL ABRAWI, MD, Department of Pediatrics, Royal Hospital; KHALID AL-THIHLI, MD, FRCPC, FCCMG, Department of Clinical Genetics, Sultan Qaboos University Hospital, Muscat, Oman. Address correspondence to Dr. R. Abdwani, Child Health Department, Sultan Qaboos University Hospital, P.O. Box 35, P C 123, Al Khod, Sultanate of Oman. E-mail: reemabd@hotmail.com

\section{REFERENCES}

1. Hull KM, Drewe E, Aksentijevich I, Singh HK, Wong K, McDermott EM, et al. The TNF receptor-associated periodic syndrome (TRAPS): emerging concepts of an autoinflammatory disorder. Medicine 2002;81:349-68.

2. Pelagatti MA, Meini A, Caorsi R, Cattalini M, Federici S, Zulian F, et al. Long-term clinical profile of children with the low-penetrance R92Q mutation of the TNFRSF1A gene. Arthritis Rheum 2011;63:1141-50.

3. Celebi-Tayfur A, Bilginer Y, Finetti M, Gattorno M, Ozen S. Different presentations in patients with tumor necrosis factor receptor-associated periodic syndrome mutations: report of two cases. Turkish J Pediatr 2013;55:78-81.

4. Aganna E, Hawkins PN, Ozen S, Pettersson T, Bybee A, McKee $\mathrm{SA}$, et al. Allelic variants in genes associated with hereditary periodic fever syndromes as susceptibility factors for reactive systemic AA amyloidosis. Genes Immun 2004;5:289-93.

5. Topaloglu R, Ozaltin F, Yilmaz E, Ozen S, Balci B, Besbas N, et al. E148Q is a disease-causing MEFV mutation: a phenotypic 


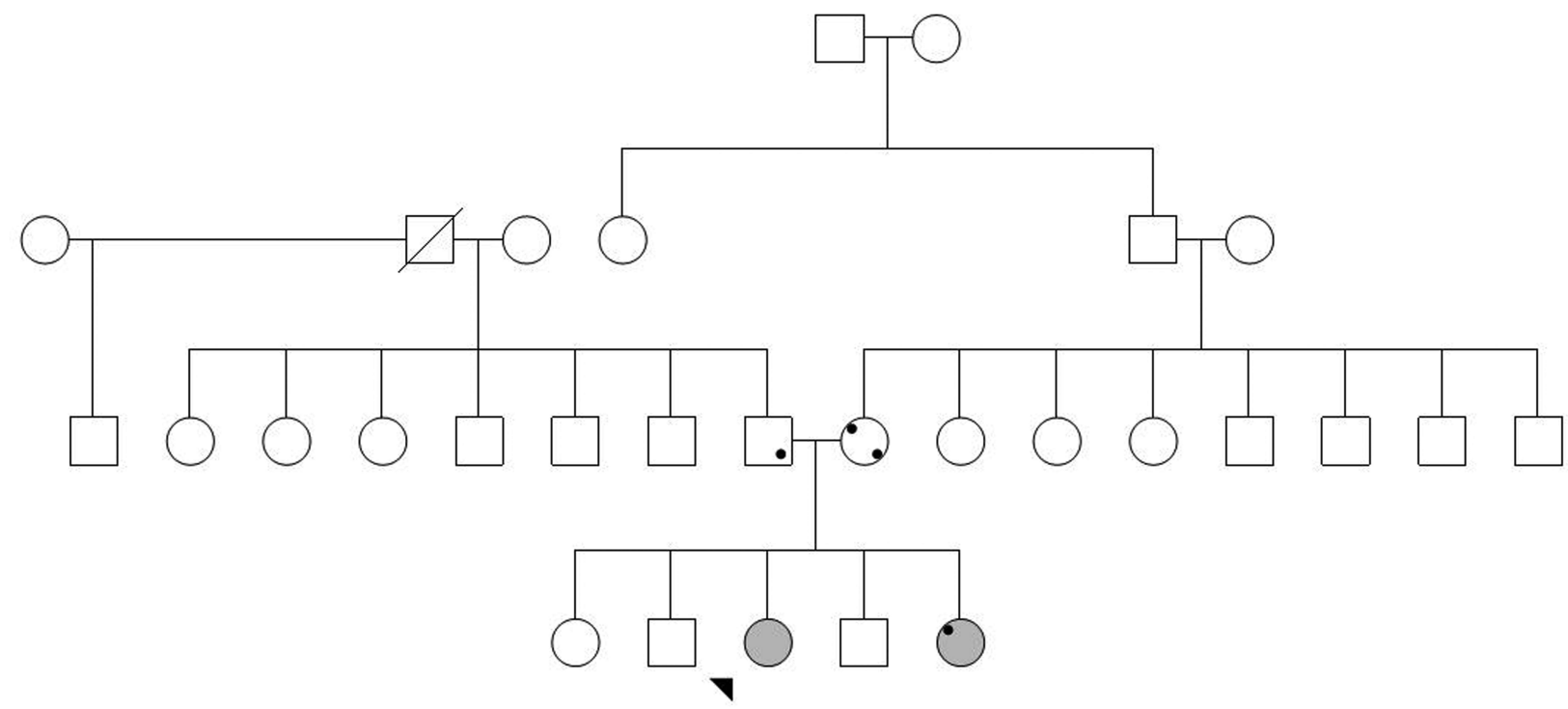

Homozygous for $\mathrm{R92Q}=\square$ Heterozygous for $\mathrm{R} 92 \mathrm{Q}=\bullet \quad$ Heterozygous for $\mathrm{E} 148 \mathrm{Q}=$

Figure 1. The family diagram, showing the distribution of both R92Q and E148Q among tested members of the family.

evaluation in patients with familial Mediterranean fever. Ann Rheum Dis 2005;64:750-2.

6. Stojanov S, Lohse P, McDermott MF, Renner ED, Kéry A, Mirakian $\mathrm{R}$, et al. Periodic fever due to a novel TNFRSF1A mutation in a heterozygous Chinese carrier of MEFV E148Q. Rheumatology 2004;43:526-527.
7. Tchernitchko D, Legendre M, Cazeneuve C, Delahaye A, Niel F, Amselem S. The E148Q MEFV allele is not implicated in the development of familial Mediterranean fever. Hum Mutat 2003;22:339-40.

J Rheumatol 2015;42:8; doi:10.3899/jrheum.141240 
DRAFT ONLY. NOT FOR CIRCULATION 2014-1240-1536 